\title{
Elaboración y Validación de un Instrumento Evaluativo para Monitorear la Adquisición de Competencias Blandas en Estudiantes de Pregrado
}

\author{
Verónica Díaz Quezada \\ mvdiaz@ulagos.cl \\ https://orcid.org/0000-0001-6428-2711 \\ Universidad de Los Lagos (ULA) \\ Osorno, Chile \\ Cecilia Sanhueza Cartes \\ csanhue@uct.cl \\ https://orcid.org/0000-0002-5998-5604 \\ Universidad Católica de Temuco (UCT) \\ Temuco, Chile.
}

Recibido: 05/03/2020 Aceptado: 15/04/2020

\section{Resumen}

El propósito de esta investigación, es mostrar la elaboración y validación por contenido en base al juicio de expertos en el tema, de un instrumento evaluativo basado en competencias blandas o transversales que permitan monitorear la adquisición de estas habilidades en educadores de párvulos, las cuales son consideradas en la actualidad por la literatura tanto nacional como internacional, imprescindibles en su formación, por su complementación con las llamadas competencias duras o genéricas. Un buen educador no solo debe reunir requisitos de tipo intelectual, sino también sociales, emocionales y personales, lo que en el ámbito educativo tiene como consecuencia cambios de dirección y orientación hacia competencias más afectivas. En el caso del alumnado de edades más pequeñas, como son los de Educacion Parvularia o Primera Infancia, los futuros docentes tendrán un papel crucial en el desarrollo de estas habilidades blandas o transversales, como por ejemplo, en la autorregulación cognitiva y emocional. Además, esas competencias sociales y emocionales que se activen a esas edades tan tempranas, jugaran un papel fundamental en el posterior desarrollo del infante.

Palabras clave: Competencias Blandas. Educación Parvularia. Instrumento Evaluativo. Educación Superior.

\section{Desenvolvimento a Validação de um Instrumento de Avaliação para Monitorar a Aquisição de Soft Skills em Estudantes de Graduação}

\footnotetext{
Resumo

O objetivo desta pesquisa é mostrar a elaboração e validação por conteúdo com base na opinião de especialistas no assunto, de um instrumento de avaliação baseado em competências transversais ou flexíveis que permitam monitorar a aquisição dessas habilidades em educadores de infância, atualmente consideradas pela literatura nacional e internacional, essenciais em sua formação, para complementação com as chamadas habilidades duras ou genéricas.Um bom professor deve não apenas atender aos requisitos intelectuais, mas também sociais, emocionais e pessoais, o que no campo educacional resulta em mudanças de direção e orientação para habilidades mais afetivas.
} 
No caso de estudantes de idades menores, como os de Educação Infantil ou Primeira Infância, futuros professores terão um papel crucial no desenvolvimento dessas habilidades suaves ou transversais como, por exemplo, na auto-regulação cognitiva e emocional. Além disso, as competências sociais e emocionais ativadas nessas idades iniciais terão um papel fundamental no desenvolvimento subsequente desenvolvimento infantil.

Palavras-chave: Soft Competencies. Educação Infantil. Instrumento Avaliativo. Educação Superior.

\title{
Elaboration and Validation of an evaluation instrument to monitor the acquisition of soft skills in undergraduate students
}

\begin{abstract}
The purpose of this research is to show the elaboration, and validation by content based on the judgment of experts in the topic of an evaluative instrument based on soft skill or transversal competences that allow monitoring the acquisition of the abilities in nursery education, which are currently considered by both national and international literature, essential for the complementation with the so-called hard or generic skills. A good teacher must not only meet intellectual, but also social, emotional and personal requirements, what in the educational field has as a consequence changes of direction and orientation towards more affective competences. In the case of students of smaller ages, such as those of nursery education o early childhood, future teachers will have a crucial role in the development of these soft or transversal skills, such as cognitive and emotional self-regulation. In addition, those social and emotional skills that are activated at these early ages, it will play a fundamental role in the further development of the infant.
\end{abstract}

Keywords: Soft Skills. Nursery Education. Evaluation Instrumen. Higher Education.

\section{Introducción}

Las habilidades del siglo XXI comprenden habilidades, capacidades y disposiciones de aprendizaje que se han identificado como necesarias para el éxito en la sociedad y los lugares de trabajo del siglo XXI por parte de educadores, líderes empresariales, académicos y agencias gubernamentales. Esto es parte de un creciente movimiento internacional que se enfoca en las habilidades requeridas para que los estudiantes dominen en preparación para el éxito en una sociedad digital que cambia rápidamente. Muchas de estas habilidades también están asociadas con un aprendizaje más profundo, que se basa en el dominio de habilidades como el razonamiento analítico, la resolución de problemas complejos y el trabajo en equipo. Estas habilidades difieren de las habilidades académicas tradicionales en que no se basan principalmente en el contenido basado en el conocimiento (Dede, 2009; Cuban, 2015; Graham, 2015). Se trata de cualidades, atributos y capacidades comunicativas que, según la literatura, no se adquieren en el ámbito académico y que no son obligatorias (pero sí deseables) para el desempeño de un trabajo en concreto, sino que se aprenden en el ámbito personal. 
Alternativamente conocidas como habilidades esenciales, habilidades del siglo XXI, habilidades sociales emocionales o habilidades para trabajar, estas "habilidades blandas" son de naturaleza interdisciplinaria. La influencia de organismos internacionales tales como World Health OrganizationWHO (2009), UNESCO (2015) y OCDE (2016) surgió en la década de los noventa en Chile, un impulso por modernizar la educación a través de la implementación del llamado currículum transversal para hacer frente a la complejidad del próximo siglo.

Es así, como en la literatura especializada, existe evidencia que el foco de análisis en la educación en Chile, es la formación inicial docente se basó en el manejo de distintos dominios, entendiendo que el mejoramiento significativo en el rendimiento académico de los estudiantes, está en directa relación con la formación del profesor que imparte la enseñanza (Cisternas, 2018; CIDE y Mineduc, 2007, Darling-Hammond y Sykes, 2003). Lo mismo se ratifica entre los resultados encontrados en las investigaciones internacionales actuales, que destacan que un buen docente no solo debe reunir requisitos de tipo intelectual, sino también sociales, emocionales y personales (Pertegai-Felices, Castejon-Costa y Martinez, 2011).

A la edad de los párvulos, los niños dependen en gran medida de los adultos que los rodean pasando un tiempo importante con sus educadoras. Por lo tanto, por definición, una parte importante de las relaciones a esta edad son jerárquicas. Las relaciones igualitarias con sus compañeros brindan a los párvulos la oportunidad de experimentar la colaboración y la competencia. Para comunicarse de manera efectiva dentro de su grupo de pares, los niños deben relacionarse con los puntos de vista de los demás, comprender a los demás y al mismo tiempo aclarar efectivamente sus propias posiciones. Estas habilidades sociales constituyen una fuente importante para su futuro bienestar social (Campbell, 2002; Denham et al., 2003) y el funcionamiento académico (Dong Hwa y Juhu, 2003).

En Chile, los jardines infantiles donde realizan la práctica profesional los educadores de párvulos son variados. Están las escuelas de párvulos, los jardines privados, Integra, JUNJI. Dada la importancia de la primera infancia, se han creado las Bases Curriculares de Educación Parvularia, generada por parte de la Subsecretaría de Educación Parvularia, el año 2018.

Existen estudios que le dan crédito a la relación existente entre el rendimiento en el trabajo y su relación con las competencias emocionales (Murga y Ortego, 2003; Boyatzis, 2006; Brotheridge y Lee, 2010); Según los autores Uitto, Jokikokko y Estola (2015), Pekrun y 
Linnenbrink-Garcia (2014), esto repercutiría en cambios tanto de orientaciones como de direcciones hacia competencias que se consideran mucho más efectivas.

Pero prácticamente no existen instrumentos evaluativos para los futuros educadores de párvulos que den cuenta, por una parte, de las competencias blandas adquiridas, y por otra, de los aspectos prácticos de su ejercicio profesional antes de egresar de los programas de formación (Coll, Taylor y Grainer, 2002; Orellana, Gorrochotegui, Lagomarsino y Mies, 2014).

En general, un estudiante promedio de una carrera de Educación Parvularia en Chile, logra ser un profesional en la medida en que aprueba las asignaturas que están su plan de estudio y que evidencian conocimiento y competencias duras. Sin embargo, existen otras habilidades que, si bien forman regularmente parte del perfil de egreso de la carrera, como se pudo comprobar en la revisión de los perfiles de egreso de varias carreras de Educación Parvularia, estás son escasamente evaluadas por las instituciones, que tienen relación con las distintas interacciones que los estudiantes efectúan durante su paso por la enseñanza superior.

De hecho, según Corporán (2014) la mitad de las actividades de aprendizaje que los maestros realizan em sus clases, no apuntan a enseñar habilidades blandas, según estudios realizados por la OECD, MICROSOFT y la Sociedad Internacional de la Información, (Corporán, 2014).

Las habilidades blandas se consideran aquellas habilidades de relación humana e interacción que no son específicas del trabajo. Son notables pero difíciles de medir. Las habilidades duras tienden a ser aquellas habilidades que son más específicas del trabajo o de la industria y más mensurables (Mitchell, 2017), por lo tanto, la posibilidad de contar con un instrumento que permita monitorear el estado de apropiación de los estudiantes respecto a las habilidades blandas o transversales que se complementan con las habilidades duras o genéricas que cada institución requiere, resulta imprescindible para las instituciones formadoras. Existe consenso con respecto a la necesidad de monitorear la progresión de estos elementos fundamentales de la formación pedagógica, por lo que crear instrumentos que no existen, instancias y modalidades de evaluación es un punto importante del diseño curricular en especial en Educación Parvularia, dado que los futuros docentes de esta área, en muchos casos serán los responsables no solo del aprendizaje curricular, sino también de la educación, desarrollo y socialización de los menores, donde las emociones y su correcto manejo y comprensión juegan un papel fundamental. 


\section{Objetivos}

De las consideraciones precedentes, el presente estudio tiene como objetivo general explicar el proceso de elaboración y validación de un instrumento evaluativo para conocer las competencias blandas de los estudiantes de Educación Parvularia de la enseñanza superior.

Los objetivos específicos son (1) Revisión de los perfiles de egreso de las carreras de Educación Parvularia según las Bases Curriculares y Marco para la Buena Enseñanza de Educación Parvularia en Chile en competencias blandas. (2) Selección y definición de las categorias de competencias blandas.

\section{Marco Teórico}

\section{Inteligencia emocional}

El tema de la inteligencia emocional ha ganado interés desde la introducción original a través de los trabajos de Salovey y Mayer (1990) y Goleman (1995). La evidencia del creciente interés en la inteligencia emocional está presente en una búsqueda del término en la base de datos multidisciplinaria de Thoreau, que arroja cientos de resultados (Dean y East, 2019). Las organizaciones interesadas en comprender el vínculo entre el desempeño organizacional y las emociones han estudiado la inteligencia emocional (Chin, Anantharaman y Tong, 2015).

Wisker y Poulis (2015) identificaron la inteligencia emocional como un componente de las habilidades blandas. Si bien existe la consideración de la inteligencia emocional en un entorno laboral positivo, Ybarra, Kross y Sanchez-Burks (2014) declararon que las emociones de un individuo en un entorno laboral negativo, podrían tener un efecto negativo en las habilidades interpersonales de la persona. Para Joseph, Jin, Newman y O’Boyle (2015), la inteligencia emocional es el vínculo entre las emociones y el rendimiento que impulsa el interés corporativo en el tema. La satisfacción laboral, la motivación, las habilidades interpersonales y la calidad de vida pueden afectar las emociones de un individuo (Siddiqui y Hassan, 2013).

De acuerdo con Serrat (2010), la inteligencia emocional describe la capacidad, habilidad o capacidad autopercibida para identificar, evaluar y manejar las emociones de uno mismo, de los demás y de los grupos. Las personas que poseen un alto grado de inteligencia emocional se conocen muy bien y también pueden sentir las emociones de los demás. Son afables, resistentes y optimistas. La inteligencia emocional se puede distinguir en dos partes de acuerdo con el rendimiento superior u ordinario de los individuos y los equipos: Inteligencia intrapersonal: ser 
consciente de sí mismo e inteligente al identificar pensamientos y emociones; También tener autogestión para hacer frente a estos pensamientos y sentimientos. Inteligencia interpersonal: ser inteligente para ser consciente de los demás e identificar sus pensamientos y sentimientos; También tiene habilidades de gestión de relaciones que son efectivas para organizar las acciones en las cooperaciones adecuadamente (Palethorpe, 2006).

Los autores Cejudo y Lopez (2017) dan cuenta de un estudio dirigido a conocer la opinión de los profesores sobre la importancia de algunas de las dimensiones que componen el dominio muestral de la inteligencia artificial, para ser un maestro altamente competente. En una muestra de 196 docentes de Educación Parvularia y Educación Básica, se evaluó la importancia de algunas de las dimensiones que componen el dominio muestral de la inteligência artificial (IE), para ser un profesor altamente competente. También se evaluó el nivel de inteligencia emocional del profesorado. Los docentes de Educación Parvularia expresaron mayor valoración de la importancia de las dimensiones de la inteligência artificial que los docentes de Educación Básica. Los docentes con alta IE mostraron un mayor reconocimiento de la importancia de las dimensiones que componen el dominio muestral de la IE para ser un profesor altamente competente que sus colegas con niveles bajos de IE.

\section{Habilidades blandas}

Según la literatura, a partir de los años noventa aparecen las llamadas habilidades o competencias del siglo XXI (Murnane y Levy, 1996; Pellegrino y Hilton, 2012; Soland, Hamilton y Stecher, 2013; Duckworth y Yeager, 2015). Luego de ello, aparece el término de soft skills o habilidades blandas (Heckman y Kautz, 2012).

Para Urhan (2018), las habilidades blandas se conocen como habilidades de las personas y contribuyen a las habilidades duras. Se pueden definir fácilmente como la capacidad de interacción con los demás y están relacionados con las emociones, los sentimientos, el instinto, es decir, el conocimiento interno. Estas habilidades pueden mejorar el desempeño laboral, las perspectivas de carrera y las relaciones individuales. Si bien son innatas y no pueden enseñarse como las habilidades duras, se pueden desarrollar y mejorar con suficiente práctica.

Aprender habilidades blandas no es un pensamiento realista porque las habilidades blandas no son las habilidades que uno puede aprender de memoria. Ya han existido en la naturaleza de casi todas las personas, y la única forma de usarlas es identificarlas y desarrollarlas a través de algunos esfuerzos: mediante el autoaprendizaje a través de algunas herramientas u 
obtener capacitación de maestros o formadores. Sin embargo, las habilidades no cognitivas pueden ser más susceptibles a la intervención directa que la capacidad cognitiva, particularmente más allá de la infancia y la primera infancia (Cunha y Heckman, 2008; Dee y West, 2011; Heckman y Kautz, 2013)

Existen diferentes definiciones de habilidades blandas, pero casi todas ellas definen en un punto la comprensión común de la naturaleza humana. Una de las primeras definiciones dadas por World Healt Organization WHO (1994) sobre habilidades para la vida es la siguiente: "son habilidades para un comportamiento positivo y de adaptación, que permite a los individuos lidiar efectivamente con las demandas y los retos de todos los días” (WHO, 1994, p.1).

Las habilidades que se definieron como básicas para el bienestar de la niñez y adolescentes fueron: la toma de decisiones, resolución de problemas, pensamiento creativo, pensamiento crítico, comunicación efectiva, habilidades para las relaciones interpersonales, empatía, manejo de las emociones, manejo del estrés, auto consciencia. Sin embargo, es importante clarificar que las habilidades sociales forman parte de las habilidades blandas, pero estas últimas están conformadas por otras categorías que puede a su vez implicar el uso de varias habilidades de diversas categorías de manera complementaria (Mangrulkar, Whitman,y Posner, 2001).

Todas las habilidades blandas se han dimensionado desde distintas perspectivas y en las definiciones expuestas solo se busca destacar una conceptualización que integra los elementos básicos de la habilidad, por tanto, no existe una definición única, ni existe una única definición de cada una de las habilidades que forman parte del conjunto. La revisión de la literatura, diferencia de las habilidades duras a las habilidades blandas, las cuales están orientadas a permitir que las personas desarrollen y exploten sus habilidades sociales, las que necesariamente deben ser complementadas con las habilidades duras.

De acuerdo a Cinque (2016), existen diferentes nombres para denominar a las habilidades blandas en algunos países de Europa. Por ejemplo, la OECD (2013) utiliza los términos 'competencias clave' y más recientemente, 'habilidades para el progreso social' (OECD, 2015). Alemania las denomina "competencias claves y general competencias" ((Schülsselkompetenzen, übergreifende; (Kompetenzen), Austria: “competencias clave" (Schüsselkompetenzen), Bélgica: “competencias transversales y competencias clave” Belgium fr: competences transversales; Belgium nl. Sleutelcompetenties). Inglaterra las denominan 
"competencias claves, competencias centrales, habilidades sociales y competencias transversales" (Key skills- Enlagand, Ireland; Core skills-Scotland; Life skills, key transferable skills, cross). Dinamarca "competencias clave" (Nøglekompetence). España: Habilidades sociales y competencias genéricas. Francia: Competencias transversales (Compétences transveresales). Italia: Competencias transversales (Competenze trasversali)

Meta Competencias (Meta competenze) y Portugal: Competencias esenciales (Competências essencias), Competencias transversales o genéricas (Compeências Transveresais ou genéricas).

Según Mujica (2015), las competencias blandas son atributos de una persona que le permiten relacionarse y comunicarse de manera efectiva con otras manifestando habilidades sociales, tanto en el contexto del trabajo como en la cotidianidad. Para Gardner (2014), estas habilidades se refieren a tener una mente disciplinada, con capacidad de síntesis, de trabajo en equipo, con presencia de liderazgo, con creatividad y ética. CIDE (2013) orienta las habilidades blandas a las aptitudes sociales de la persona. Las refiere capacidades de trabajo en equipo y de comunicación entre otras.

A diferencia de las habilidades duras, técnicas o cognitivas, a las que Iturralde (2003) las define como destrezas técnicas que permiten desempeñar tareas específicas y que se logran a través del proceso formativo disciplinar y entrenamiento en el mismo ejercicio de las funciones que se requieren. Por otra parte, para este autor, las habilidades blandas tienen que ver con habilidades actitudinales necesarias para tener una buena relación con las demás personas, escuchar, hablar, comunicarse, liderar, estimular, delegar, analizar, entre otras. (Iturralde, 2003).

Al agrupar todas estas definiciones se puede confirmar el valor que tienen las habilidades blandas, sobre todo si se enfocan específicamente en el área de la Educación Parvularia, para lograr una buena relación profesor-infante.

Para efectos de esta investigación, compartimos la definición de competencias blandas de Murti (2014), como habilidades que no son técnicas y que se han determinado necesarias en el ambiente de trabajo, son transversales en las competencias del individuo y están más relacionadas a la personalidad, actitud y comportamiento de cada persona. 


\section{Investigaciones de competencias blandas internacionales}

Según The Automation Readiness Index (2018), la educación y la capacitación deben tomarse en serio y deben desarrollarse estrategias a largo plazo para manejar los desafíos que la automatización traerá en el futuro. Todos los estudiantes, es decir, las futuras fuerzas laborales deben ser educados y entrenados en cuanto a las habilidades que las computadoras nunca tienen. Se deben tomar precauciones a nivel país para adaptarse a las necesidades futuras. Por ejemplo, con respecto al informe, se comprueba que Corea del Sur se destaca como el líder que agrega habilidades blandas al plan de estudios. Alemania, Singapur y Japón toman los siguientes tres rankings después de Corea del Sur. El World Economic Forum (2019) realizó una encuesta a 56.000 jóvenes entre 15 y 35 años, para conocer para conocer las actitudes y las habilidades, y el impacto de la tecnología en el futuro del trabajo en seis países: Indonesia, Malasia, Filipinas, Singapur, Tailandia y Vietnam. Los jóvenes de la Asociación de jóvenes de las naciones del sudeste asiático ASEAN, valoraron más las habilidades blandas que las habilidades duras, y creen que son más competentes en las habilidades blandas. Cuando se les pregunta qué habilidades creen que serán más valiosas en el futuro, valoran más las habilidades blandas como la inteligencia emocional, la resistencia y adaptabilidad que, en habilidades duras, generalmente consideradas como "STEM": ciencia, tecnología, ingeniería y matemáticas

Dentro de los instrumentos administrados a los estudiantes para conocer su inteligencia emocional, se elaboró el Trait Meta-Mood Scale 48 (TMMS-48) de los autores Salovey, Mayer, Goldman, Turvey y Palfai (1995), el Schutte Self Report Inventory (SSRI) de Schutte, Malouff, Hall, Haggerty, Cooper, Golden y Dornheim (1998). A continuación, se elaboraron los test Multifactor Emotional Intelligence Scale (MEIS) y el Emotional Intelligence Test conocido como MSCEIT, ambos de los autores Mayer, Caruso y Salovey (1999, 2002), respectivamente.

Por su parte, la OECD (2015) inició un estudio sobre habilidades sociales y emocionales SSES, en estudiantes entre 10 y 15 años, con el objetivo de proporcionar información sobre las condiciones y prácticas que fomentan u obstaculizan el desarrollo de estas habilidades. Este estudio comenzó a mediados de 2017 y durará tres años; el trabajo de campo principal tendrá lugar en 2019 y los hallazgos se publicarán en 2020.

La OECD Learning Compass 2030 distingue entre tres tipos diferentes de habilidades: habilidades cognitivas y metacognitivas; habilidades sociales y emocionales; y habilidades físicas y prácticas. Indican que las habilidades sociales y emocionales, como la empatía, la 
autoconciencia, el respeto por los demás y la capacidad de comunicarse, se están volviendo esenciales a medida que las aulas y los lugares de trabajo se vuelven más étnica, cultural y lingüísticamente diversa (OECD, 2018)

Continuando en el ámbito profesional internacional, Palomera, Fernández-Berrocal y Brackett (2008) aseveran que recientemente se ha comenzado a analizar la relación entre la inteligencia emocional y el bienestar del docente. En Chile, no existe tradición sobre la evaluación de estas competencias, sin embargo, en países anglosajones destaca Estados Unidos, donde se han realizado importantes investigaciones al respecto, invirtiendo alrededor de 50 billones de dólares cada año en formación del profesorado, de la cual, gran parte se centra en capacidades sociales y emocionales (Cherniss, 2000).

Los autores Yan, Yinghong, Man, Whiteside y Tsey (2019), evaluaron la viabilidad de un curso de bienestar australiano en el contexto de la capacitación explícita en habilidades blandas en una universidad china, en un piloto con 29 estudiantes de segundo año. Los resultados muestran claramente que los participantes encontraron relevante y aceptable tanto el contenido de la capacitación como el enfoque de aprendizaje social participativo. La capacitación tuvo un impacto positivo estadísticamente significativo en los resultados evaluados de las habilidades blandas de las cualidades humanas. Se destacan las implicaciones de los hallazgos para el interés actual en capacitar a estudiantes universitarios chinos en el espíritu de innovación y empresa.

El entrenamiento en competencias duras como se les ha denominado a las destrezas cognitivas necesarias para el desarrollo de una profesión, fueron y en algunos casos siguen siendo el interés central de las instituciones de educación superior en Chile, como es el caso de la Prueba de Selección Universitaria PSU como requisito de ingreso a las instituciones de educación superior, el Programa para la Evaluación Internacional de Estudiantes PISA, entre otras, llegando a considerarse necesarias para obtener éxito a nivel profesional (Bassi, Busso, Urzua, y Vargas, 2012). Lo cual a juicio de Singer et al. (2009), no necesariamente es así. En algunos casos se han considerado necesarios para obtener un buen trabajo e incluso éxito profesional (Bassi et al., 2012). Sin embargo, este aspecto es contradictorio, ya que también se ha encontrado que los resultados de estas pruebas no son necesariamente predictivos de éxito profesional (Singer et al., 2009). 
No obstante, a juicio de Guerra (2019), son pocas las investigaciones que se han interesado en evaluar las competencias blandas en estudiantes universitarios y su relación con futuro el desempeño laboral. Autores como John (2009), Singer et al. (2009), Fernández y Tapia (2012), Albarrán y González (2015), Gómez, Manrique-Lozada y Gasca-Hurtado (2015), han realizado estudios exploratorios en este campo, pero dada su importancia en la formación de educadores y sobre todo de infantes, no es suficiente.

Esto se explicaría porque al revisar la literatura, en muchos casos el desarrollo de las habilidades blandas se relaciona con características propias de la personalidad del individuo, por lo que teóricamente dificultaría la posibilidad de poder modificarlas a través del entrenamiento (Moss y Tilly, 1996; Brunello y Schlotter, 2011; Nitonde, (2014). Autores como Cunha y Heckman (2007) al respecto argumentan que, si las habilidades blandas son consideradas como aspectos de la personalidad, entonces solo podría lograrse un desarrollo de estas, primero al tenerlas los propios educadores de párvulos para luego poder entrenarlas desde edades muy tempranas, es decir, durante la infancia a sus estudiantes.

Según el World Economic Forum WHO (2018), la comparación de la demanda de habilidades 2018 frente a 2022, son mostradas en la Figura 1

Figura 1. Comparing skills demand, 2018 vs 2022, top ten

\begin{tabular}{|c|c|}
\hline Today, 2018 & Trending, 2020 \\
\hline $\begin{array}{l}\text { - Analytical thinking and } \\
\text { innovation } \\
\text { - Complex problem-solving } \\
\text { Critical thinking and } \\
\text { analysis } \\
\text { - } \quad \text { Active learning and } \\
\text { learning strategies } \\
\text { - } \quad \text { Creativity, originality and } \\
\text { initiative } \\
\text { - } \quad \text { Attention to detail, } \\
\text { trustworthiness } \\
\text { - } \quad \text { Emotional intelligence } \\
\text { Reasoning, problem- } \\
\text { - } \quad \text { Lelving and ideation } \\
\text { influence } \\
\text { - Co-ordination and time } \\
\text { management }\end{array}$ & $\begin{array}{l}\text { Analytical thinking and } \\
\text { innovation } \\
\text { Active learning and learning } \\
\text { strategies } \\
\text { Creativity, originality and } \\
\text { initiative } \\
\text { Technology design and } \\
\text { programming } \\
\text { Critical thinking and analysis } \\
\text { Complex problem-solving } \\
\text { Leadership and social } \\
\text { influence } \\
\text { Emotional intelligence } \\
\text { Reasoning, problem-solving } \\
\text { and ideation } \\
\text { Systems analysis and } \\
\text { evaluation }\end{array}$ \\
\hline
\end{tabular}

Fuente: Future of Jobs Survey 2018, World Economic Forum

Del mismo modo, el World Economic Forum afirma que las 10 habilidades que disminuirán el 2022 son: Manual dexterity, endurance and precision; Memory, verbal, auditory and spatial abilities; Management of financial, material resources; Technology installation and 
maintenance; Reading, writing, math and active listening; Management of personnel; Quality control and safety awareness; Co-ordination and time management; Visual, auditory and speech abilities; Technology use, monitoring and control.

De acuerdo al informe de tesis doctoral en la Universidad de Kentucky, las cinco habilidades blandas más valoradas son: la integridad, la capacidad de comunicación, la responsabilidad, las habilidades sociales y el liderazgo (Wayne, 2019).

Un estudio reportado por Young (2018) llevado a cabo en el Centro de Investigación de Stanford concluye que el $85 \%$ del éxito profesional depende del desarrollo de las llamadas habilidades blandas y solo el $15 \%$ está vinculado a destrezas y conocimientos técnicos adquiridos a lo largo del tiempo de su formación académica, es decir, habilidades duras o genéricas, que se acreditan con las actas de notas, los registros académicos, los títulos obtenidos, los certificados de capacitación, las constancias de idiomas aprendidos, los grados académicos (Boyatzis, 2006), entre otros. Aunque muchas personas poseen inherentemente este tipo de habilidades, cualquiera puede trabajar para desarrollarlas. En cambio, dentro de las habilidades blandas son importantes los buenos modales, el optimismo, el sentido común, el sentido del humor, la empatía y la capacidad de colaborar y negociar. Además, una persona con un alto nivel de inteligencia emocional tiene buenas habilidades de comunicación y gran capacidad para trabajar en equipo.

En Jordania presentaron Groh, Krishnan, McKenzie, y Vishwanath (2016) un programa para evaluar las habilidades blandas que utiliza tres elementos: evaluaciones a través de juegos de roles, ejercicios grupales y entrevistas para indagar sobre las habilidades. Por su parte, Escocia realizó un proyecto en el año 2011 en este mismo ámbito, cuyo propósito era medir y evaluar las habilidades blandas. De acuerdo a sus resultados, el liderazgo y la conciencia del contexto, fueron las habilidades claves tanto para el desarrollo de las personas, el trabajo y el empleo (Kechagias, 2011).

En España, la investigación más reciente la realizaron Balaguer, Pujol y Grell (2018), cuyo objetivo fue presentar y analizar el desarrollo del aprendizaje de los estudiantes que cursan la formación inicial de maestros de Educación Parvulararia. Para ello, consideraron cuestionamientos tales como que, en la carrera, los estudiantes tienen que adquirir diferentes competencias que les permitan convertirse en buenas personas y buenos profesionales. Pero también cuestionaron ¿cuál es el proceso a través del que los estudiantes pasan a asumir las 
competencias comunicativas y el pensamiento crítico que les permitirán ser maestros? ¿Cómo autorregulan los estudiantes sus aptitudes lingüísticas, ya sea las orales y escritas? ¿Están conscientes con su propio aprendizaje en esta materia y son además críticos de ella? A través de estas reflexiones, se plantea el estudio de los procesos meta cognitivos que permiten la cristalización de los conocimientos y las habilidades lingüísticas. Los autores prevén que la investigación dure 2 años. La metodología responde a un modelo longitudinal mixto para lograr informaciones sobre la percepción de los estudiantes de grado de Educación Parvularia, acerca el desarrollo de su competencia lingüística y comunicativa. En este artículo concretamente se pretende describir el diseño de la investigación, el instrumento elaborado y los primeros resultados de naturaleza cualitativa de los que se desprende la necesidad de mejorar los procesos de reflexión de los estudiantes de grado de Educación Infantil o Educación Parvularia.

En América Latina, el Banco Interamericano de Desarrollo, efectuó entre el 2008 y el 2010 una investigación que buscó observar sistemáticamente las habilidades blandas en Chile, Argentina y Brasil con la finalidad de conocer la relación existente entre la educación en la escuela y la educación para los jóvenes en el contexto laboral. Cabe hacer notar que esta investigación tuvo un importante impacto (Bassi et al., 2012).

\section{Investigaciones en Educación Parvularia en Chile}

En Chile, los educadores de párvulos o de primera infancia, son agentes especializados en la educación de los niños de entre 0 a 6 años. Una investigación en el ámbito de la Educación Parvularia, es la medición que se realizó sobre la calidad de vida desde la perspectiva de la salud y su relación en el ámbito laboral a 156 de estos profesionales de la educación. De acuerdo a los resultados que hacen referencia al uso de un cuestionario, se encontraron relaciones altamente positivas en la función física, es decir, que el estado de salud encontrado era compatible con su trabajo. También se percibe una relación del rol emocional con el ausentismo y dependencia laboral, además de una estrecha relación entre la vitalidad y el número de niños a cargo (Arteaga, Hermosilla, Mena y Contreras, 2018).

El Instituto de Estudios Avanzados en Educación CIAE de la Universidad de Chile, realizó una investigación para la Organización de Estados Americanos y la Subsecretaría de Educación Parvularia de Chile, cuyo objetivo era la caracterización de las prácticas de liderazgo en Educación para la Educación Parvularia en Iberoamérica, con especial énfasis en el trabajo 
que se desarrolla en jardines infantiles chilenos. Los hallazgos del estudio, se relacionan con una síntesis del estado del arte sobre el liderazgo ejercido en Educación Parvularia, tema escasamente abordado en Chile y en el resto de Iberoamérica, según los autores del estudio; aun cuando aseguran, que existe en Chile mayor preocupación por la calidad de la Educación Parvularia; también se realiza una amplia descripción del sistema de Educación Parvularia chileno, enfatizando el rol directivo y liderazgo en el contexto chileno (CIAE, 2018-2019).

\section{Metodología y Procedimientos}

\section{Elaboración del instrumento evaluativo}

La confección del instrumento se realizó en cuatro etapas.

\section{Etapa1}

La primera consistió en la revisión de los perfiles de egreso de las carreras de Educación Parvularia y su relación con las Bases Curriculares y Marco para la Buena Enseñanza de Educación Parvularia en Chile, para extraer de cada uno de ellos la relación con las habilidades blandas o transversales que se caracterizan por ayudar a entender mejor un contexto e influir en el.

\section{Etapa 2:}

Se revisaron acuciosamente las competencias relacionadas con los perfiles de egreso en las carreras de Eduación Parvularia en Chile y su intersección, para llegar a las definiciones internacionales de las competencias más utilizadas en el siglo XXI. De ellas se eligieron las competencias blandas que se reconocen internacionalmente como las más importantes, que coinciden además con las que forman parte de la formación del futuro educador de párvulos, las que posteriormente constituirían la base del instrumento evaluativo. De esta forma, se seleccionaron las siguientes cinco categorias de habilidades o competencias blandas, que se indican a continuación en la Figura 2. 
Figura 2. Categorías de competencias blandas

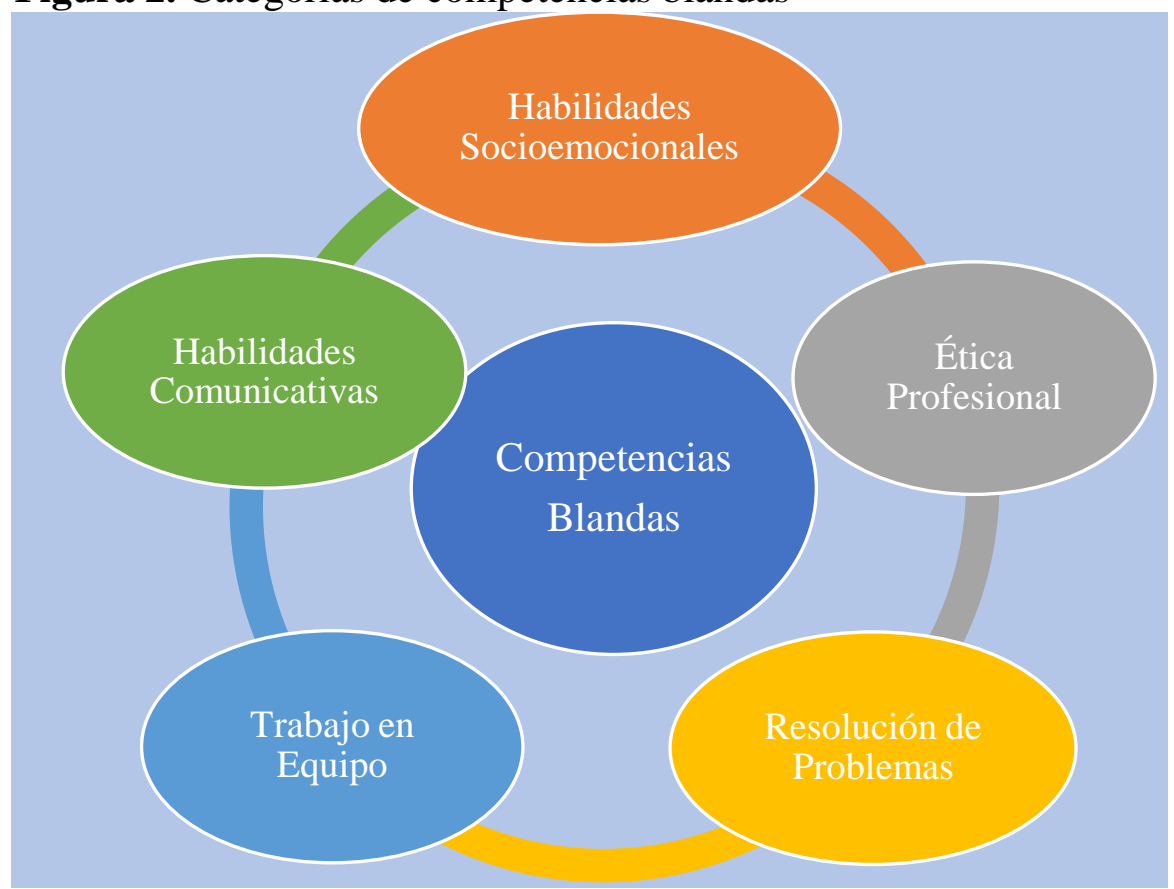

Fuente: Elaborada por las autoras

Etapa 3: Se definieron las categorías que formarán parte del instrumento evaluativo

\section{Definición de las categorías de competencias blandas}

\section{Habilidades Socioemocionales}

La perseverancia, sociabilidad y curiosidad son habilidades socioemocionales que, como su nombre lo indica, están estrechamente relacionadas con las emociones, las cuales se encuentran presentes en todos nuestros comportamientos y son las encargadas de motivar, energizar y dirigir tanto el pensamiento como la conducta (West, 2016).

\section{Ética Profesional}

La ética profesional, considera que los maestros están sujetos a un conjunto de normas comunes de conducta profesional que existen principalmente para garantizar que brinden la más alta calidad de servicio público y ejerzan la autoridad que les otorga la sociedad de manera responsable. Típicamente articuladas en un código de ética profesional o documento afín, las normas de profesionalismo ético están destinadas a guiar la conducta de los profesionales con un propósito social específico: mantener la confianza pública en la profesión (Maxwell y Schwimmer, 2016). 


\section{Resolución de problemas}

Las habilidades para resolver problemas son un conjunto de habilidades blandas para usar en asuntos difíciles, inesperados o complicados que surgen en el lugar de trabajo. Las habilidades blandas para resolver problemas generalmente permiten que un individuo se prepare para los problemas antes de que sucedan. La resolución de problemas involucra aspectos de varios otros conjuntos de habilidades blandas, ya que requiere elementos de comunicación, creatividad, fiabilidad, toma de decisiones, liderazgo y análisis exhaustivo (Contessa, 2019).

\section{Trabajo en equipo}

El trabajo en equipo responde a las personas que trabajan juntas en un entorno cooperativo para lograr objetivos comunes del equipo mediante el intercambio de conocimientos y habilidades El trabajo en equipo exitoso se basa en la sinergia existente entre todos los miembros del equipo que crean un ambiente donde todos están dispuestos a contribuir y participar para promover y nutrir un ambiente positivo y efectivo entorno de equipo. Los miembros del equipo deben ser lo suficientemente flexibles como para adaptarse a entornos de trabajo cooperativo donde los objetivos se alcanzan mediante la colaboración y la interdependencia social en lugar de objetivos competitivos individualizados (Luca y Tarricone, 2001)

\section{Habilidades comunicativas}

Corresponden a una sólida habilidad que asegura una comunicación clara dentro del grupo. Alienta a los miembros del equipo a compartir información, habla por todo y valora las contribuciones de todos los miembros. Además, requiere saber plantear de manera adecuada lo que se quiere decir, considerando el contexto y el medio que usará para hacerlo (Alles, 2006).

\section{Etapa 4}

En esta fase se elaboró el instrumento evaluativo consistente en un cuestionario de opinión en relación a las cinco categorías de competencias blandas con 41 afirmaciones medidas en una escala Likert de 5 instancias de respuesta: (5) muy de acuerdo, (4) de acuerdo, (3) indiferente, (2) en desacuerdo y (1) muy en desacuerdo, en la cual el encuestado debe marcar una sola respuesta. Se trata de un solo instrumento que puede ser aplicado en forma anónima y 
con fines de investigación, a los propios estudiantes educadores de párvulos (autoevaluación).

La composición del instrumento se muestra a continuación en los Cuadros 1 al 5.

\section{Instrumento para el monitoreo de las habilidades blandas}

Cuadro 1: Habilidades Socioemocionales

\section{Categoria: Habilidades Socioemocionales}

Es capaz de mostrarse amable en momentos de estrés

Sabe establecer un diálogo entre las prioridades del infante y las propiedades técnicas

Tiene una vida personal feliz fuera de la Universidad

Se cuida a sí misma/o en momentos de estrés

Se preocupa y cuida a sus compañeras/os en momentos de estrés

Tiene la capacidad de ponerse en el lugar de la otra persona

Es capaz de comunicar sus sentimientos y emociones a otros

Las decisiones no las asume con responsabilidad

Mantiene una relación más bien regular con todos en su carrera

Manifiesta reacciones de emoción frente a ciertas situaciones

Administra su tiempo en función de prioridades

Cuadro 2: Ética Profesional

\section{Categoría: Ética profesional}

Comprende su actuar de acuerdo con el sentido ético que la educación demanda

Es puntual y hace las cosas a tiempo

Tiene capacidad para tomar iniciativas

Se considera confiable y cumple las promesas que asume

Establece vínculos de confianza y afecto

Se relaciona con su entorno laboral en forma armoniosa

Manifiesta aceptación de todo tipo de diversidad

Cuadro 3: Resolución de problemas

\section{Categoría: Resolución de problemas}

Piensa lógicamente frente a una situación difícil

Encuentra soluciones ante una situación difícil

Es capaz de resolver un problema de manera efectiva y rápida

Es capaz de analizar una información y definir una solución o estrategia de acción

Tiene capacidad para organizarse y hacer una gran cantidad de tareas en poco tiempo

Considera que puede idear una solución para un tema de su trabajo que se encuentra estancado

Si tiene que actuar ante un inconveniente, simplemente lo evade

Ante un problema, piensa más en la solución que en el problema mismo 
Cuadro 4: Trabajo en equipo

Categoría: Trabajo en equipo

Le agrada trabajar sola/o

Escucha y aprecia las opiniones de todos los miembros del curso

Apoya y enseña a sus compañeros con menores conocimientos

Impone sus ideas por sobre los demás miembros del curso

Cumple con los acuerdos establecidos por el grupo del curso

Respeta las opiniones de los demás aunque sean diferentes a las suyas

Es incluyente con los miembros del curso y se mantiene al margen

Comunica toda la información posible al resto de sus compañeros para el logro de los objetivos en

común

Cuadro 5: Habilidades comunicativas

Categoria: Habilidades comunicativas

Considera que se expresa adecuadamente de forma verbal

Es capaz de jerarquizar asuntos relativos al trabajo y comunicarlos de forma oportuna

Considera que me expresa adecuadamente de forma escrita

Sabe escuchar a los demás

Comprende las necesidades de los demás

Tiene sentido del humor y lo utiliza en momentos acertados

Considera que expresa sus ideas en su curso de manera efectiva

Considera que se expresa adecuadamente de forma verbal

\section{Validación del instrumento evaluativo}

Los expertos en medición han enfatizado que la validez no es una característica inherente de una medida en sí misma, sino más bien una característica de una medida con respecto a un uso final particular (AERA, APA, NCME, 1999/2014). Por lo tanto, las diferentes medidas, con sus ventajas y limitaciones únicas, son válidas de manera diferencial dependiendo no solo de sus propiedades psicométricas, sino también de su aplicación prevista.

El cuestionario de opinión basado en competencias blandas fue validado por contenido mediante el juicio de ocho expertos en el tema (Skjong y Wentworth, 2001), todos académicos universitarios. Se les hizo llegar una tabla de operacionalización de las variables, que se iniciaba con las definiciones de cada una de las cinco categorías consideradas en esta investigación. Un cuestionario con 50 preguntas con las cuales debían relacionar la pertenencia a la categoría de trabajo en equipo, habilidades comunicativas, ética professional, resolución de problemas y habilidades sócioemocionales.

Se consideró un $85 \%$ de congruencia entre sus respuestas, para aceptar como válida la categoría y las preguntas asociadas a ellas. Finalmente conformaron el instrumento evaluativo 41 de ellas. (Hyrkäs, Appelqvist-Schmidlechner y Oksa, 2003) 
Una vez piloteado, se elaborará la versión final y se calculará su consistencia interna para ser aplicado a los estudiantes de Educación Parvularia del octavo semestre de formación, dado que se encuentran en periodo de práctica profesional insertos en un equipo de trabajo en diversas instituciones de Educación Parvularia, tales como, salas cuna, jardines infantiles, escuelas u otro, en un par de universidades de Chile y en dos regiones del país.

\section{Conclusiones y Comentarios Finales}

El instrumento evaluativo se encuentra en una fase previa a su primera aplicación formal a varias cohortes de estudiantes. Se espera que el instrumento permita contar con información acerca del grado de desarrollo de las distintas competencias blandas necesarias para trabajar mejor en el contexto pedagógico; entendiendo este contexto tanto en las relaciones que se producen al interior del aula en la Universidad, como también entre pares en el curso.

Con este instrumento evaluativos como insumo, la institución formadora de profesores de párvulos, estarán en mejores condiciones de dar cuenta de la labor orientada tanto a la formación intelectual como la pedagógica y profesional, con el fin de contribuir a que el país tenga los docentes que necesita para alcanzar una verdadera educación de calidad para todos.

Entre los motivos que explican por qué deben trabajarse en el aula las competencias blandas, coincidimos con Hargreaves (2000) en el sentido en que en la actualidad las familias tienen más dificultad para centrarse en la educación de sus hijos/as, debido a las exigencias tanto laborales como sociales del diario vivir. Esto ha hecho que los docentes en muchos casos sean los responsables no solo del aprendizaje curricular, sino también de la educación, desarrollo y socialización de los menores, donde las emociones y su correcto manejo y comprensión juegan un papel fundamental. Por tanto, el rol docente implica una importante carga de trabajo emocional, tanto en la comprensión de las emociones ajenas como en la regulación de las emociones propias para facilitar la calidad de las relaciones sociales dentro del contexto escolar.

Además, el alumnado adapta su comportamiento al tipo de actitud, claves emocionales y afectivas y conductuales que los docentes transmiten, por lo tanto, dada la importancia del desarrollo de las competencias blandas, sumada a la escasa evaluación que de ellas existe, lo que se puede verificar tanto en la literatura nacional como internacional, se vuelve imprescindible elaborar un instrumento evaluativo que permita monitorear la adquisición efectiva de las competencias blandas que complementan a las competencias duras. 
En un entorno de acreditación de Educación Parvularia en educación superior centrado en los resultados, la implementación de una evaluación, seguida de un análisis cuantitativo de los resultados, es probable que sea el primer y el siguiente paso para muchas universidades. Tal evaluación de las habilidades blandas de los estudiantes podría entonces, utilizarse para proporcionar una idea del potencial de las respuestas de los y estudiantes de Educación Parvularia de educación superior, con respuestas reflexivas y multifacéticas que podrían proporcionar una hoja de ruta inicial para un plan integral, para abordar la necesidad de habilidades blandas.

\section{Referencias}

Albarrán, A. y González, C. (2015). Introduciendo habilidades no cognitivas en liceos técnicos chilenos. Una Evaluación Social (Tesis de pregrado) Universidad de Chile, Santiago, Chile.

Alles. M. (2006). Diccionario de preguntas: Gestión por competencias. Buenos Aires: Granica, S.A.

Arteaga, P, Hermosilla-Ávila, P, Mena, C. y Contreras, S. (2018). Una mirada a la calidad de vida y salud de las educadoras de párvulos. Ciencia \& Trabajo, 20(61), 42-47.

American Educational Research Association, American Psychological Association, National Council on Measurement in Education (AERA/APA/NCME) (1999). Standards for educational and psychological testing. Washington, DC: American Educational Research Association.

American Educational Research Association, American Psychological Association, National Council on Measurement in Education (AERA/APA/NCME) (2014). Standards for educational and psychological testing. Washington, DC: American Educational Research Association.

Balaguer, M. Pujol, M., y Grell, M. (2018). Desarrollo del pensamiento crítico a través de la competencia comunicativa en la educación superior: una propuesta reflexiva. Innoeduca. International Journal of Technology and Educational Innovation, 4(1), 54-61.

Bassi, M., M. Busso, S. Urzua, y Vargas, J. (2012). Desconectados: Habilidades, educación y empleo en América Latina. USA: BID - Fondo de Cultura Económica.

Boyatzis, R. E. (2006). An overview intentional change theory from a complexity perspective. Journal of Management Development, 25(7), 607-623. doi: 10.1108/02621710610678445

Brotheridge, C. M. y Lee, R. T. (2010). Development and validation of the emotional labour scale. Journal of Occupational and Organizational Psychology,76(3), 365-379. doi: 10.1348/096317903769647229

Brunello, G. y Schlotter, M. (2011). Non cognitive skills and personality traits: Labour market relevance and their development in education \& training systems. IZA Discussion Paper No. 5743. Recuperado de http://ftp.iza.org/dp5743.pdf

Campbell, S. B. (2002). Behavior problems in preschool children. New York: Guilford Press. 
Cejudo, J. y Lopez, M.L. (2017) Importancia de la inteligencia emocional en la práctica docente: un estudio con maestros. Psicología Educativa, 23(1), 29-36. doi.org/10.1016/j.pse.2016.11.001

Centro de Investigación Avanzada en Educación CIAE (2018-2019). Caracterización del liderazgo y el rol de los /as directores/as en establecimientos para la primera infancia. Santiago: Universidad de Chile.

CIDE (2013) Habilidades blandas fundamentales para el desarrollo personal. Revistas Grupo Educar, 186, 22-23. Recuperado de http://www.cide.cl/documentos/Revista_educar_Habilidades_blandas_MJValdebenito.pdf.

CIDE y MINEDUC (2007). Estado del arte de la investigación y desarrollo en educación en Chile. Santiago: Ministerio de Educación.

Cinque, M. (2016). "Lost in translation". Soft skills development in European countries. Tuning Journal for Higher Education, 3(2), 389-427. doi: 10.18543/tjhe-3(2)-2016pp389-427

Cisternas, T. (2018). La investigación sobre formación docente en Chile. Territorios explorados e inexplorados. Calidad en la Educación, 35,131-164. doi: 10.31619/caledu.n35.98

Coll, R.K., Taylor, N. y Grainger, S. (2002). Assessment of work based learning: Some lessons from the teaching profession. Asia-Pacific Journal of Cooperative Education, 3(2), 5-12.

Contessa C. (2019, July 24). Are you adding your soft skills to your resume? Here's how. Recuperado de https://www.careercontessa.com/advice/resume-soft-skills/.

Corporán, M. (2014). Competencias blandas. Congreso internacional Aprendo 2014, INFOTED, República Dominicana.

Cuban, L. (2015). Content vs. skills in high schools - 21st century arguments echo 19th century conflicts. Recuperado de https://larrycuban.wordpress.com/2015/11/03/content-vs-skillsin-high-schools-21st-century-arguments-echo-19th-century-conflicts/

Cunha, F. y Heckman,J. (2007). The technology of skill formation. American Economic Review, 97(2),31-47. doi: 10.1257/aer.97.2.31

Cunha, F., Heckman, J. J. (2008). Formulating, identifying and estimating the technology of cognitive and noncognitive skill formation. Journal of Human Resources, 43(4), 738-782. doi: $10.3368 / \mathrm{jhr} .43 .4 .738$

Cherniss, C. (2000). Emotional intelligence: What it is and why it matters. In Proceedings of the Annual Meeting of the Society for Industrial and Organizational Psychology, New Orleans, LA. Recuperado de http://www.talentsmart.com/media/uploads/pdfs/eq-what-itis.pdf.

Chin, S., Anantharaman, R., y Tong, D. (2015). Emotional intelligence and organisational performance: A framework. Global Business \& Management Research: An International Journal, 7(2), 37-43.

Darling-Hammond, L. y Sykes, G. (2003). Wanted: a national teacher supply policy for education: the right way to meet the "highly qualified teacher" challenge. Education Policy Analysis Archives, 11(33),1-55.

Dean, S. y East, J. (2019). Soft skills needed for the 21st century workforce. International Journal of Applied Management and Technology,18(1), 17-32. doi:10.1016/j.paid.2016.11.018 
Dee, T. S., y West, M. R. (2011). The non-cognitive returns to class size. Education Evaluation and Policy Analysis, 33(1), 23-46. doi.org/10.3102/0162373710392370

Dede, C. (2009). Comparing frameworks for "21st century skills". In J. Bellanca \& R. Brandt (Eds.), 21st Century Skills: Rethinking How Students Learn (pp. 51-75). Bloomington, IN: Solution Tree Press.

Denham, S. A., Blair, K.A., De Mulder, E., Levitas, J., Sawyer, K., Auerbach- Major, M., y Queenan, P. (2003). Preschool emotional competence: Pathway to social competence. Child Development, 74(1), 238-256. doi:10.1111/1467-8624.00533

Dong Hwa, C., y Juhu, K. (2003). Practicing social skills training for young children with low peer acceptance: A cognitive-social learning model. Early Childhood Education Journal, 31(1), 41-46. doi: 10.1023/A:1025184718597

Duckworth, A. y Yeager, D. (2015). Measurement matters: Assessing personal qualities other than cognitive ability for educational purposes. Educational Researcher, 44(4), 237-251. doi: 10.3102/0013189X15584327

Fernández, J. y Tapia, V. (2012). Emprendimiento y desarrollo de competencias blandas por alumnos de ingeniería comercial Universidad de la Serena. Revista Universitaria Ruta, 14, 41-56.

Gadner, H. (2014). Inteligencias múltiples. La teoría en la práctica. Barcelona, España: Editorial Paidos.

Goleman, D. (1995). Emotional intelligence: Why it can matter more than IQ. New York, NY: Bantam Dell.

Gómez, M. C., Manrique- Lozada, B., Gasca-Hurtado, G.P. (2015). Propuesta de evaluación de habilidades blandas en ingeniería de software por medio de proyectos universidad-empresa. Revista Educación en Ingeniería, 10(19), 131-140. doi: https://doi.org/10.26507/rei.v10n19.549

Graham, S. (2015). Preparing for the 21st Century: Soft skills matter. Huffington Post. Recuperado de https://www.huffpost.com/entry/preparing-for-the-21st-ce_b_6738538.

Groh, M. N., Krishnan, D. McKenzie, y Vishwanath, T. (2016). The impact of soft skills training on female youth employment: Evidence from a randomized experiment in Jordan. IZA Journal of Labor \& Development, 5(9), 1-23. doi:10.1186/s40175-016-0055-9.

Guerra, S. (2019). Una revisión panorámica al entrenamiento de las habilidades blandas en estudiantes universitarios. Psicología Escolar e Educacional, 23, versión On-line ISSN 0718-4565. doi:0000-0001-5367-5069

Hargreaves, A. (2000). Mixed emotions: teachers' perceptions of their interactions with students Teaching and Teacher Education, 16(8), 811-826. doi: 10.1016/S0742-051X(00)00028-7

Heckman, J. y Kautz, T. (2012). Hard evidence on soft skills. Labour Economics, 19(4), 451464. doi: 10.1016/j.labeco.2012.05.014.

Heckman, J. J., Kautz, T. (2013). Fostering and measuring skills: Interventions that improve character and cognition. In Heckman, J. J., Humphries, J. E., Kautz, T. (Eds.), The myth of achievement tests: The GED and the role of character in American life (pp. 341-430). Chicago, IL: University of Chicago Pres. 
Hyrkäs, K., Appelqvist-Schmidlechner, K. y Oksa, L. (2003). Validating an instrument for clinical supervision using an expert panel. International Journal of Nursing Studies, 40(6), 619-625. doi:10.1016/s0020-7489(03)00036-1

Ybarra, O., Kross, E., y Sanchez-Burks, J. (2014). The "big idea" that is yet to be: Toward a more motivated, contextual, and dynamic model of emotional intelligence. Academy of Management Perspectives, 28(2), 93-107. doi.org/10.5465/amp.2012.0106

Iturralde, E. (2003). Habilidades para un futuro exitoso. Ecuador: Worldwide Inc.

John, J. (2009). Study on the nature of impact of soft skills training programme on the soft skills development of management students. Pacific Business Review, 19-27. Recuperado de https://papers.ssrn.com/sol3/papers.cfm?abstract_id=159133.

Joseph, D., Jin, J., Newman, D., y O’Boyle, E. (2015). Why does self-reported emotional intelligence predict job performance? A meta-analytic investigation of mixed. Journal of Applied Psychology, 100(2), 298-342. doi.org/10.1037/a0037681

Kechagias, K. (2011). Teaching and assessing soft skills. $1^{\text {st }}$ Second Chance School of Thessaloniki, Neapolis: MASS Project.

Luca, J., y Tarricone, P. (2001). Does emotional intelligence affect successful teamwork? In Kennedy, G., Keppell, M. McNaught, C. \& Petrovic, T. (Eds.), Meeting at the Crossroads. Proceedings of the 18th Annual Conference of the Australian Society for Computers in Learning in Tertiary Education. (pp. 365-376). Melbourne: Biomedical Multimedia Unit, The University of Melbourne.

Mangrulkar, L., Whitman, C.V. y Posner, M. (2001). Enfoque de habilidades para la vida para un desarrollo saludable de niños y adolescentes. Washington, DC: Organización Panamericana de Salud.

Mayer, J. D., Caruso, D. R., y Salovey, P. (1999). Emotional intelligence meets traditional standards for an intelligence. Intelligence, 27 (4), 267-298. doi.org/10.1016/S01602896(99)00016-1

Mayer, J. D., Caruso, D. R. y Salovey, P. (2002). Emotional Intelligence Test (MSCEIT): User's Manual. Toronto, Canada: MHS Publisher.

Maxwell, B., y Schwimmer, M. (2016). Professional ethics education for future teachers: A narrative review of the scholarly writings. Journal of Moral Education, 45(3), 1-18. doi:10.1080/03057240.2016.1204271

Mitchell, A. (2017). Determining the skills gap for new hires in management: Student perceptions vs employer expectations. International Journal for Innovation Education and Research, 5(6), 139-147. Recuperado de https://www.ijier.net/index.php/ijier/article/view/732

Moss, P. y Tilly, C. (1996). "Soft" skills and race: An investigation of black men's employment problems. Work and Occupations, 23(3), 252-276. doi: 10.1177/0730888496023003002

Mujica, J. (2015). Habilidades blandas, No. 1. ¿Qué son las habilidades blandas y cómo se aprenden? Recuperado en https://educrea.cl/wp-content/uploads/2016/02/DOChabilidades-blandas.pdf

Murga, J. y Ortego, E. (2003). La importancia de la inteligencia emocional en el funcionamiento de las organizaciones. Encuentros en Psicologia Social, 1(4), pp. 79-82. 
Murnane, R.J. y Levy, F. (1996). Teaching the new basic skills: Principles for educating children to thrive in a changing economy. New York, NY: The Free Press.

Murti, A. B. (2014), Why soft skills matter. IUP Journal of Soft Skills, VIII(3), 32-36.

Nitonde, R. (2014). Soft skills and personality development. Proceedings in National Level Seminar on Soft Skills and Personality Development. Shri Shivaji College, Parbhani (MS) India.

OECD (2013). Skilled for life? Key findings from the survey of adult skills. OECD Skills Studies. Paris: OECD Publishing.

OECD (2015). Skills for social progress: The power of social and emotional skills. Paris, Francia: OECD Publishing.

OECD (2016). Global competency for an inclusive world. París, Francia: OCDE.

OECD (2018). The Future of education and skills. Education 2030. París, Francia: OCDE

Orellana, P., Gorrochotegui, A. Lagomarsino, R. y Mies, R. (2014). Desarrollo de un instrumento para monitorear la adquisición de habilidades de trabajo en equipo en estudiantes de carreras de educación. Investigación y Postgrado, 29(1), 103-128.

Palethorpe, M. (2006). Are you emotional but intelligent- or are you emotionally intelligent? Engineering Management, 16(19, 11-13. doi: 10.1049/em:20060101

Palomera, R., Fernández-Berrocal, P. y Brackett, M.A. (2008). La inteligencia emocional como una competencia básica en la formación inicial de los docentes: algunas evidencias. Revista Electrónica de Investigación Psicoeducativa, 6(2), 437-454.

Pekrun, R. y Linnenbrink-Garcia, L. (2014). International handbook of emotions in education. New York: Routledge.

Pellegrino, J.W. y Hilton, M.L. (2012). Education for life and work: Developing transferable knowledge and skills in the 21st century. Washington, DC: The National Academy of Sciences.

Pertegai-Felies, M.A., Castejón, J. y Martinez, M.A. (2011). Competencias socioemocionales en el desarrollo profesional del maestro. Educación XX1,14(2), 237-260.

Salovey, P., y Mayer, J. D. (1990). Emotional intelligence. Imagination, Cognition and Personality, 9, 185-211. doi.org/10.2190/DUGG-P24E-52WK-6CDG

Salovey, P., Mayer, J.D., Goldman, S.L., Turvey, C. y Palfai, T.P. (1995). Emotional attention, clarity and repair: exploring emotional intelligence using the trait meta-mood scale. In Pennebaker, J. (Ed.) Emotion, Disclousure and Healt. Washington, D.C.: American Psychological Association.

Schutte, N. S., Malouff, J. M., Hall, L. E., Haggerty, D. J., Cooper, J. T., Golden, C. J. y Dornheim, L. (1998). Development and validation of a measure of emotional intelligence. Personality and individual differences, 25(2), 167-177. doi: 10.1016/S0191-8869(98)000014.

Serrat, O. (2010). Understanding and developing emotional intelligence. Washington, DC: Asian Development Bank.

Siddiqui, R., y Hassan, A. (2013). Impact of emotional intelligence on employees turnover rate in FMCG organizations. Pakistan Journal of Commerce \& Social Sciences, 7(2), 394-404.

Singer, M., R. Guzman, y Donoso, P. (2009). Entrenando competencias blandas en jóvenes. Escuela de administración: Pontificia Universidad Católica de Chile. Recuperado en 
http://www.inacap.cl/tportal/portales/tp90b5f9d07o144/uploadImg/File/PDF/Entrenando_ Competencias_Blandas_en_Jovenes.pdf

Skjong, R. y Wentworth, B. (2001, June 17-22). Expert judgement and risk perception. Proceedings of Eleventh the International Offshore and Polar Engineering Conference, Stavanger, Norway.

Soland, J., Hamilton, L.S. y Stecher, B.M. (2013). Measuring 21st century competencies: Guidance for educators. Santa Monica, CA: RAND Corporation.

The Automation Readiness Index. (2018). Who is ready for the coming wave of automation? The Economist Intelligence Unit Limited. Supported by AAB. Recuperado de http://www.automationreadiness.eiu.com/static/download/PDF.pdf ).

Uitto, M., Jokikokko, K. y Estola, E. (2015). Virtual special issue onteachers and emotions in teaching and teacher education (TATE) in 1985-2014. Teaching and Teacher Education, 50, 124-135. doi.org/10.1016/j.tate.2015.05.008

UNESCO (2015). Transversal competences in education policy and practice. París, Francia: UNESCO.

Urhan, B. (2018). Soft skill: making use of the human nature to have a job in the age of technology. Social Sciences Studies Journal, 4(28), 6298-6304. doi.org/10.26449/sssj.1113

Wayne, K. (2019). Soft skills: old \& new (Theses and Dissertations-Educational Policy Studies and Evaluation) 65. Recuperado en https://uknowledge.uky.edu/epe_etds/65

West, R. M. (2016). Should non-cognitive skills be included in school accountability systems? Preliminary evidence from California's CORE districts. Brookings Economic Studies: Evidence Speaks Reports, 1(13). Recuperado de https://www.brookings.edu/wpcontent/uploads/2016/07/EvidenceSpeaksWest031716.pdf.

Wisker, Z., y Poulis, A. (2015, January 27). Emotional intelligence and sales performance. A myth or reality? International Journal of Business \& Society, 16(2), 185-200. doi: 10.33736/ijbs.563.2015

World Health Organization WHO (1994). Division of mental health. Life skills education for children and adolescents in schools. Pt. 1, Introduction to life skills for psychosocial competence. Pt. 2, Guidelines to facilitate the development and implementation of life skills programmes, 2nd rev. Geneva: World Health Organization. Recuperado en https://apps.who.int/iris/handle/10665/63552

World Economic Forum WHO (2009). Preventing violence by developing life skills in children and adolescents. Geneva, Switzerland: World Economic Forum

World Economic Forum WHO (2018). The future of education, according to experts at Davos. Geneva: Switzerland: World Economic Forum

World Economic Forum WHO (2019). ASEAN Youth Technology, Skills and the Future of Work. Geneva: World Education Forum.

Yan, L., Yinghong, Y., Man L., Whiteside, M, y Tsey, K. (2019). Teaching "soft skills" to university students in China: the feasibility of an Australian approach. Educational Studies, 45(2), 242-258.

Young. R. (2018). Soft skills: The primary predictor of success in academics, career and life. Recuperado en https://pairin.com/2018/07/13/soft-skills-primary-predictor-successacademics-career-life/. 